\title{
Crisis del empleo y polarización de las trayectorias laborales. El caso de los adultos jóvenes en Cataluña
}

\author{
Joan Miquel Verd \\ (IET) \\ joanmiquel.verd@uab.cat \\ Martí López-Andreu \\ University of Manchester. Manchester Business School \\ marti.lopezandreu@mbs.ac.uk
}

Universitat Autònoma de Barcelona. Departament de Sociologia. Centre d'Estudis

Sociològics sobre la Vida Quotidiana i el Treball (QUIT) e Institut d'Estudis del Treball

Recibido: 13-10-2014

Aceptado: 23-07-2015

\section{Resumen}

El artículo presenta los resultados de una investigación cuyo objetivo es analizar de qué modo la crisis económica y del empleo en España ha afectado a las trayectorias laborales de la población menor de 40 años. Las trayectorias durante este período de crisis se analizan desde dos puntos de vista. En primer lugar, descriptivamente, construyendo una tipología de trayectorias y analizándola comparativamente con la población de más de 40 años. En segundo lugar, causalmente, identificando aquellos factores que permiten explicar los diferentes tipos de trayectorias hallados. Estos análisis permiten comprobar hasta qué punto se ha dado, durante la crisis, una polarización entre las trayectorias de las personas pertenecientes a las cohortes más jóvenes y aquellas de mayor edad y, por otro lado, si también, dentro de las cohortes más jóvenes, se ha dado un progresivo distanciamiento en el tipo de trayectorias desarrolladas. Los resultados obtenidos muestran que la "polarización intergeneracional» se da en menor medida de la que se suele defender en la literatura internacional, mientras que la «polarización intrageneracional» no es propiamente una polarización, sino una segmentación en tres tipos. En cuanto al análisis causal, se comprueba que las variables clásicas de segmentación en el mercado laboral español han seguido marcando las trayectorias laborales durante la crisis. Factores como el sector de actividad o el origen social han tenido también un efecto causal.

Palabras clave: trayectorias laborales; crisis económica; polarización; segmentación del mercado laboral; inestabilidad laboral; transiciones laborales; España; Cataluña 
Abstract. Employment crisis and polarisation of labour market trajectories. The case of young adults in Catalonia

The article presents the results of a research aimed at analyzing how economic and employment crisis in Spain has affected the labour trajectories of the population under 40 years. The trajectories during this period are analyzed from two points of view. First, descriptively, building a typology of trajectories and analyzing it in comparison with the population of more than 40 years. Second, causally, identifying the factors that explain the different types of trajectories found. These analyses assess to what extent has been, during the period of crisis, a polarization between the trajectories of persons belonging to the younger cohorts and those belonging to the older ones. On the other hand, the analyses also assess whether it has been a progressive gap within the younger cohorts on the type of trajectories developed. The results show that the «inter-generational polarisation» is given in a lesser extent than is often defended in the international literature, while the "intra-generational polarisation" is not really a polarisation, but a segmentation into three types. As to causal analysis, it is found that the classical variables of segmentation in the Spanish labour market have continued to influence the labour trajectories during the crisis. Factors such as sector or social origin have had also a causal effect.

Keywords: labour trajectories; economic crisis; polarization; labour market segmentation; labour instability; labour transitions; Spain; Catalonia

\begin{aligned} & \multicolumn{2}{c}{ Sumario } \\ & Introducción 3. Los efectos de la crisis en las \\ & trayectorias laborales de los adultos \\ & 1. La noción de polarización y su jóvenes. Factores de diferenciación \\ & laborales 4. Datos y metodología \\ & 2. Polarización del empleo y de las 5. Resultados \\ & trayectorias laborales en España, 6. Conclusiones \\ & un fenómeno de largo recorrido Referencias bibliográficas \end{aligned}

\section{Introducción}

El presente artículo presenta una aproximación a los efectos que la crisis económica y del empleo en España, iniciada en $2007^{1}$, ha tenido sobre las trayectorias laborales. Es conocido que España se caracteriza, a nivel europeo, por unas elevadas tasas de temporalidad y flexibilidad contractual, así como por unas altas tasas de desempleo. Este modelo de empleo especialmente flexible ha tendido a generar trayectorias laborales inestables, que se habían concentrado hasta el inicio de la crisis en los grupos más vulnerables del mercado de trabajo: jóvenes, mujeres, inmigrantes y trabajadores con escasa cualificación. Sin embargo, está aún por comprobar el modo en que la crisis ha afectado a

1. Desde el punto de vista del empleo, la recesión empezó en España durante el cuarto trimestre de 2007. 
esta población laboralmente vulnerable y si sus efectos se han hecho sentir sobre aquellos trabajadores que, durante los años de crecimiento económico, habían evitado las situaciones de inestabilidad.

La literatura a nivel internacional sugiere que, durante la presente crisis del empleo, se están profundizando los fenómenos de polarización que se habían iniciado en los años ochenta. Así, la crisis estaría produciendo dos efectos diferentes sobre las trayectorias de los trabajadores cuya inserción laboral ya se produjo en un entorno de flexibilidad. El primer efecto sería un alejamiento relativo (como mínimo, durante los años que llevamos de crisis) respecto a las condiciones de trabajo de los individuos de mayor edad, y el segundo, un incremento de las diferencias internas entre los trabajadores de edad más joven que pertenecen a una misma generación. Sin embargo, la virulencia de la crisis en España podría hacer pensar en un acercamiento (a la baja) entre los diferentes segmentos. En este sentido, cabe preguntarse si la crisis del empleo en España ha introducido algún elemento diferenciador respecto a la evolución de las trayectorias laborales o si "simplemente» ha acentuado las tendencias existentes con anterioridad a la crisis.

El artículo concentra su análisis en la población hasta los 40 años. Son estas cohortes más jóvenes las protagonistas del empleo temporal en el período anterior a la crisis y, supuestamente, serían las más afectadas por el empeoramiento de las condiciones de trabajo durante aquella. El análisis realizado permite caracterizar a esta población en términos de continuidad y discontinuidad de sus trayectorias laborales, e identifica qué factores y características personales y del mercado de trabajo han afectado a su desarrollo. Para ello, se aplicará una perspectiva longitudinal, lo cual hace imprescindible el uso de datos de tipo panel o, en su defecto, de carácter retrospectivo. La aplicación de esta perspectiva longitudinal en el marco actual de crisis del empleo estaba aún pendiente de hacerse en el contexto español.

Dada la inexistencia de encuestas longitudinales a nivel estatal, se ha optado por utilizar la base de datos proveniente del Panel de Desigualdades $(\mathrm{PaD})$ de la Fundació Jaume Bofill (2012), que ofrece datos únicamente para Cataluña. Se trata de una encuesta que permite reconstruir las trayectorias laborales de los encuestados para una secuencia de años y que, además, ofrece información especialmente relevante para nuestros objetivos. Con estos datos, se reconstruyen las trayectorias laborales entre los años 2007 y 2011, ambos incluidos, para la población de entre 20 y 65 años. Las trayectorias obtenidas para el período indicado se analizan desde dos puntos de vista. En primer lugar, descriptivamente, construyendo una tipología de trayectorias y analizándola comparando la población de entre 20 y 40 años con la que se sitúa entre los 41 y los 65 años. En segundo lugar, causalmente, y concentrando el análisis en la submuestra de individuos cuya edad se sitúa hasta los 40 años, identificando aquellos factores que permiten explicar los diferentes tipos de trayectorias hallados.

El artículo se divide en cinco apartados. En primer lugar, se realiza un breve repaso a la noción de polarización y a su aplicación al análisis de las trayectorias laborales. En segundo lugar, se presentan las principales características 
de la polarización y segmentación del empleo y de las trayectorias laborales en España. Seguidamente, se caracterizan las dinámicas del empleo durante la crisis económica, prestando especial atención a la situación de la población más joven. En cuarto lugar, se describen la base de datos utilizada y los análisis llevados a cabo. En quinto lugar, se presentan los resultados obtenidos, $y$, finalmente, en el sexto apartado, se presentan las conclusiones principales.

\section{La noción de polarización y su extensión al análisis de las trayectorias laborales}

Una de las consecuencias de la crisis económica actual en todos los países occidentales ha sido la polarización del empleo. Con diferentes matices, y utilizando diversos indicadores, los trabajos desarrollados hasta el momento (Eurofound, 2013, Autor, 2014; Carrasco et al., 2014) han hallado que, en la estructura del empleo de las economías desarrolladas, se reducen los puestos de trabajo de "calidad media» (de salario y cualificación medios, y una cierta estabilidad), y, en cambio, aumentan los puestos de trabajo con menor estabilidad, cualificación y salario, lo que podría denominarse «empleo de mala calidad». Esta tendencia se daba ya antes de la crisis económica actual (Goos et al., 2009; Cedefop, 2011; Häusermann y Schwander, 2012; Moreno-Galbis y Sopraseuth, 2014) ${ }^{2}$, pero se habría incrementado fuertemente como consecuencia de la crisis. El debate se ha dado en el momento de identificar las causas de esta tendencia, puesto que se ha atribuido a factores tan diversos como el cambio tecnológico, la globalización de los mercados y las decisiones en políticas de empleo tomadas en los países occidentales.

En este debate en torno a las causas de la polarización, Emmenegger et al. (2012) se posicionan afirmando que los factores institucionales y las políticas públicas son elementos determinantes. De hecho, abogan por el uso del término dualización para referirse a las políticas de empleo y de protección social que estarían causando, como uno de sus resultados (outcomes), la situación de polarización entre diferentes perfiles de trabajadores y trabajadoras (2012: 11-12). Estos mismos autores plantean que una de las dimensiones empíricas a tener en cuenta en este debate es la de la biografía de empleo (employment biography), como modo de entender los efectos que los cambios en las políticas públicas están teniendo sobre las personas.

Esta preocupación por los aspectos longitudinales y biográficos contrasta fuertemente con las investigaciones al uso sobre la polarización del empleo, en que el análisis tiene un carácter estático (cuando se vincula directamente al estudio de la segmentación del mercado de trabajo) o, en el mejor de los casos, está basado en el análisis conjunto de una serie sucesiva de fotografías de situación, aunque sin tomar en consideración el grado en que los individuos

2. Aunque ciertos autores afirman que, en el caso español, este proceso de polarización no se ha dado durante el último período de crecimiento económico. Véase Bohomme y Hospido (2013) o Carrasco et al. (2014). 
tienen posibilidad de transitar entre los diferentes segmentos identificados. Este hecho no deja de llamar la atención, puesto que, en la formulación teórica inicial de la teoría de la segmentación laboral (Doeringer y Piore, 1971; Edwards, 1975), se hacía un fuerte énfasis en la dimensión longitudinal, destacando las dificultades existentes para salir del segmento con peores condiciones laborales. En este modelo teórico, el segmento primario y el secundario se conciben como cerrados el uno al otro, de modo que las trayectorias laborales de los trabajadores se desarrollarían enteramente en un solo segmento (Tomlinson y Walker, 2012).

Únicamente aquellas investigaciones que se han preocupado por el fenómeno del atrapamiento de la población joven en la inestabilidad laboral han puesto el acento en la dimensión longitudinal de la polarización del empleo, aunque no necesariamente utilizan explícitamente la noción de trayectoria (Hernanz, 2003; Scherer, 2004; Berton et al., 2011; García-Pérez y MuñozBullón, 2011; Bruno et al., 2012). En estos casos, el objetivo que se persigue es conocer hasta qué punto las carreras laborales que se inician en condiciones de precariedad e inestabilidad laboral se desarrollan con características similares a lo largo de los años. Aunque los resultados no son concluyentes, posiblemente debido a los diversos métodos utilizados y a la limitación de las bases de datos, se comprueba que, en todos los países analizados, se da en mayor o menor medida el fenómeno del atrapamiento. Solamente algunas de las personas con mayor nivel de formación consiguen superar la desventaja inicial asociada a los empleos descualificados y temporales situados en el segmento secundario.

En el planteamiento que presentamos en este artículo, la noción de trayectoria es central. De hecho, tomamos un enfoque muy cercano al de Emmenegger et al. (2012) y Häusermann y Schwander (2012), aunque las preguntas que se pretenden responder se orientan al nivel individual, mucho más que al institucional. Así, se estudiarán aquellas características individuales que permiten explicar, en interacción con los factores institucionales y las políticas de empleo desarrolladas en España, que ciertos trabajadores y trabajadoras desarrollen trayectorias caracterizadas por la temporalidad contractual, el desempleo y la inactividad, frente a otros que desarrollen trayectorias caracterizadas por la estabilidad. Todo ello teniendo en cuenta que España ha sido uno de los países de la Unión Europea que ha padecido (y sigue padeciendo) los efectos de las crisis económica con mayor virulencia.

Este tipo de análisis no se ha desarrollado aún en el contexto español, y menos aún desde una perspectiva sociológica. Los escasos estudios que han abordado el estudio de la polarización del empleo en España desde una perspectiva longitudinal lo hacen desde un enfoque exclusivamente económico. Así, una variable de tanta relevancia como el origen social familiar se ha dejado de lado, o incluso se analiza solo de forma indirecta el peso que el nivel de estudios tiene en la situación laboral observada. Ello se debe, como se ha indicado en la introducción, a la inexistencia de encuestas longitudinales a nivel estatal, lo cual obliga en muchas ocasiones al uso de registros administrativos. 


\section{Polarización del empleo y de las trayectorias laborales en España, un fenómeno de largo recorrido}

Aunque no existe unanimidad al respecto ${ }^{3}$, autores como Martín y Gibert (2006), Bernardi y Garrido (2008) u Oesch y Rodríguez (2010) indican que, durante el último período de crecimiento económico, la creación de empleo en España ha mostrado una tendencia a la polarización ocupacional, en la que han aumentado simultáneamente ambos extremos de la estructura de empleo. Por otro lado, tal como muestran los estudios comparados (Cedefop, 2011), España ha sido el país europeo que, durante el período 2000-2008, ha mantenido la reserva más elevada de empleo de baja cualificación. Basándose en el análisis de la estructura ocupacional, Bernardi y Garrido (2008) destacan la importancia numérica de este tipo de trabajo, que se ha mantenido a lo largo de los años a pesar de las posibilidades de que ciertos grupos (como hombres, universitarios y los que llevan poco tiempo en el desempleo) han tenido para salir del mismo. Esta dinámica se puede relacionar con el tipo de empleo creado en España durante la expansión económica, basado en el turismo y la construcción (Banyuls y Recio, 2012).

Las bases institucionales y políticas de esta polarización del empleo pueden hallarse en las sucesivas reformas flexibilizadoras que, desde mediados de la década de 1980, se han impuesto como medida de lucha contra el desempleo (Recio, 1997: 169-171; Banyuls et al., 2009). Estas medidas han generado un modelo de empleo que, por un lado, muestra una enorme sensibilidad frente a los ciclos de actividad económica (Koch, 2006; Banyuls et al., 2009; Muñoz de Bustillo y Antón, 2012) y, por otro, presenta una elevada segmentación, con grupos especialmente inestables en el mercado de trabajo (Bernardi y Garrido, 2008; Bernardi y Martínez-Pastor, 2010).

El uso masivo de contratos temporales por parte de las empresas desde inicios de los años noventa, junto con la diferente protección entre el empleo indefinido y el temporal, han servido para que algunos autores destaquen la «segmentación generacional» del mercado laboral español. Esta cuestión se ha abordado aludiendo al modelo insider-outsider (Lindbeck y Snower, 2001), argumentando que el mercado laboral español se caracteriza por un segmento cuyos trabajadores se insertaron antes de la década de los noventa, los insiders, que poseen contratos indefinidos y un elevado grado de protección, y un segundo segmento de trabajadores más jóvenes, los outsiders, sometidos a una forma de contratación mucho más flexible, con escasa protección y también una fuerte presencia de desempleo (Ferreiro et al.; 2004; Simó, 2008). Sin embargo, los análisis empíricos más recientes (Häusermann y Schwander, 2012; Lehwess-Litzmann, 2014) matizan mucho este esquema, poniendo de manifiesto las malas condiciones laborales de algunos de los teóricos insiders.

No obstante, es cierto que los segmentos más inestables han estado ocupados tradicionalmente por jóvenes, inmigrantes y mujeres, perfiles que, hasta

3. Véanse los trabajos ya mencionados de Bohomme y Hospido (2013) o Carrasco et al. (2014). 
la crisis económica, eran mayoritarios en los servicios no cualificados y en la construcción. Estos segmentos inestables y menos cualificados han sido también los protagonistas tanto del espectacular crecimiento de empleo que se produjo en España hasta el año 2007, con más de 7,5 millones de puestos de trabajo creados entre 1996 y 2007 (ILO, 2011), como de la extraordinaria destrucción posterior de empleo, hasta el punto de que se puede afirmar que ha sido el país europeo en que los efectos de la crisis económica sobre el mercado laboral han sido más acentuados (ILO, 2011).

Cuando esta situación se ha analizado longitudinalmente, se destacan las dificultades existentes para abandonar el segmento más inestable del mercado laboral. Las trayectorias de precariedad identificadas se caracterizan por una frecuencia muy baja de transiciones de la contratación temporal a la indefinida e incluso por una frecuencia elevada de transiciones de la contratación temporal a la inactividad o al desempleo en lugar de hacia el contrato indefinido (Hernanz, 2003; García-Pérez y Muñoz-Bullón, 2011). Estas trayectorias han estado asociadas, durante el período previo a la crisis económica, a unas determinadas características sociodemográficas. Así, para el periodo 2001-2006, se ha observado que las trayectorias inestables se vinculan a una fuerza de trabajo primordialmente joven, femenina y con bajo nivel educativo (Verd y López-Andreu, 2012). Por otro lado, también puede afirmarse que, en general, durante los años previos a la crisis económica actual, las trayectorias de inserción lineales que desembocaban en una contratación indefinida han ido perdiendo peso y, por el contrario, han ido ganando peso aquellos itinerarios de inserción laboral precaria (Casal et al., 2006; Serracant, 2010).

Estas características generales de los procesos de inserción laboral y de los itinerarios de trabajo durante los primeros años de presencia en el mercado laboral español podrían considerarse un ejemplo nacional más de la tendencia a la desestandarización de las trayectorias laborales que las dinámicas del empleo y el origen social provocan entre algunos miembros de las cohortes más jóvenes (Brueckner y Mayer, 2005; Fenton y Dermott, 2006; Mills et al., 2008). Sin embargo, lo que parece haber ocurrido en España durante los años previos a la crisis económica es una polarización de las trayectorias laborales, en consonancia con la ya mencionada polarización del mercado laboral. La particularidad del caso español se halla en el hecho de que, para un importante volumen de trabajadores, su inestabilidad inicial difícilmente se corrige con el transcurso de los años (Toharia et al., 2001; Cebrián, 2008; Verd y López-Andreu, 2012).

Un ejemplo de estas dificultades para salir de las situaciones de inestabilidad queda recogido en la investigación realizada por Castillo y López Calle (2007). Estos autores, a través de historias de vida y de una explotación cualitativa, muestran la emergencia de trayectorias laborales caracterizadas por la inestabilidad y de transiciones entre formación y empleo marcadas por la demanda empresarial de flexibilidad. Aunque estas dinámicas afectan tanto a las trayectorias obreras como a las más profesionales, estos mismos autores ponen de manifiesto la existencia de fuertes desigualdades entre las cohortes jóvenes, lo cual da como resultado una relación con el mercado de trabajo marcada por 
una polarización de posiciones laborales. En este mismo sentido, Alonso y Fernández (2008) indican que la construcción de la juventud en los últimos años se ha estado desarrollando en el marco de un proceso de polarización de ingresos, entre grupos de profesionales y técnicos y otros grupos marcados por posiciones más débiles que tratan de mantener unos empleos cada vez más precarios. En estos últimos colectivos, se produciría, tal como indica Santos (2006), una conexión entre el desempleo y el empleo flexible, y un ajuste permanente, complejo e incierto, entre las pretensiones de estabilidad y los requerimientos de flexibilidad del mercado laboral.

\section{Los efectos de la crisis en las trayectorias laborales de los adultos jóvenes. Factores de diferenciación}

Como se ha indicado en los apartados anteriores, la polarización de las trayectorias laborales entre determinados perfiles sociales debe relacionarse con el modelo de empleo existente tanto antes como después de la crisis. Evidentemente, la inestabilidad se ha acrecentado en el marco de la destrucción de puestos de trabajo que genera la crisis económica y de empleo. La cuestión es si la crisis económica ha puesto en acción nuevos factores de diferenciación en las trayectorias o si, básicamente, ha agravado la situación de los colectivos que más padecían la inestabilidad.

Los numerosos trabajos que han analizado, a nivel internacional y desde un punto de vista transversal, los efectos de la crisis económica sobre la población más joven (Bell y Blanchflower, 2011; De Lange et al., 2014; Heyes, 2014; entre muchos otros) alertan, en primer lugar, de la brecha que se está produciendo entre los perfiles más jóvenes y la población de mayor edad, con unas condiciones de empleo cada vez más diferenciadas y, en segundo lugar, de las diferencias internas que se observan dentro de las generaciones más jóvenes. Tomando una perspectiva longitudinal, Scarpetta et al. (2010) o Genda et al. (2010) advierten del peligro de que las diferencias intrageneracionales no solo no se corrijan con los años, sino que se incrementen, especialmente entre los jóvenes ocupados con mayor nivel académico y aquellos en situación de desempleo con niveles educativos más bajos. Este fenómeno se ha dado ya en ocasiones anteriores, en que han entrado en juego procesos de acumulación de desventajas o de "efecto cicatriz» (scarring effect) (véanse, a modo de ejemplo, Kwon et al., 2010; Hillmert, 2011; Skans, 2011; Kelly et al., 2012). Obviamente, la información disponible hasta el momento no permite identificar la magnitud de estos efectos durante la crisis económica actual, por lo que los análisis realizados hasta ahora no dejan de ser parciales. Además, la crisis no ha concluido y, en los años más recientes, los recortes en gasto público parecen estar afectando a nuevos perfiles de trabajadores (Ortiz y Cummins, 2013; Rocha y Negueruela, 2014).

Los estudios existentes para el contexto español son todos de carácter transversal, probablemente debido a la ya indicada escasez de buenos datos de carácter longitudinal. Estos trabajos (ILO, 2011; Prieto, 2012; Rocha, 2012; 
Santos y Martín, 2012) muestran que los principales afectados por la destrucción de empleo son jóvenes, mujeres, inmigrantes y, en general, trabajadores poco cualificados de los sectores de la construcción y la hostelería; aunque, en los años más recientes, sectores como la educación, los servicios sociales y las administraciones públicas también han perdido empleo (Rocha y Negueruela, 2014). Estos mismos datos transversales ponen de manifiesto que la destrucción de puestos de trabajo se ha dado principalmente entre las personas nacidas a partir de los años 1971-1975 y que entraron en el mercado laboral a inicios de la década de 1990. Es por ello que, más que hablar de jóvenes, debería hablarse de adultos jóvenes. Fueron estas mismas cohortes las protagonistas del incremento de la temporalidad durante los años previos a la crisis.

Es interesante destacar que los datos de carácter transversal también muestran que un elevado nivel de estudios permite evitar en cierta medida las situaciones de desempleo, aunque no las de empleo temporal (Fundación CYD, 2014). Este hecho supone una continuidad respecto a lo observado en el período anterior a la crisis, en que un elevado nivel educativo ya había dejado de tener el papel de protección frente a la inestabilidad (Toharia et al., 2001; García-Montalvo, 2008; Santamaría, 2012). De acuerdo con Ortiz (2010), ni siquiera la sobreeducación permitiría a los jóvenes asegurarse un puesto de trabajo estable, puesto que la fuerte segmentación del mercado laboral los mantendría «atrapados» en la temporalidad.

El grado de relación entre el nivel de estudios y la situación en el mercado laboral es un punto controvertido en la literatura que ha tratado el caso español. Mientras Cachón (2008) y Recio (2009) están de acuerdo con la escasa conexión entre tener un nivel de estudios elevado y la posición en el mercado laboral, de modo que los itinerarios hacia la vida adulta serían cada vez más dependientes del origen social, Carabaña (2004) defiende, refiriéndose a los titulados universitarios, que una credencial universitaria depara las mismas oportunidades laborales a todos aquellos que la poseen. Navarro (2013), en una investigación centrada únicamente en titulados en estudios superiores de Cataluña, afirma también, aunque de modo algo más matizado, que las características de origen social no impactan en el tipo de inserción laboral, dado el mismo nivel académico.

Sin embargo, algunos datos indican que la expansión de los niveles educativos superiores entre la población española puede haber causado su pérdida de efectividad como mecanismo de ascenso social (Martínez Celorrio y Marín, 2010), lo cual pondría de manifiesto la importancia de otros capitales, como el cultural o el relacional, en las trayectorias laborales. Además, en un contexto de escasez de empleo como el actual, la movilización de todo tipo de recursos familiares y personales, en interacción con los educativos (Carabaña, 1997), puede ser claramente una necesidad. Es por ello que, en nuestro análisis, se tendrá en cuenta el origen social familiar como factor relevante en el desarrollo de las trayectorias laborales. Ello permitirá obtener una imagen más ajustada de la influencia de las credenciales educativas en la polarización de las trayectorias de empleo en un contexto de crisis. 


\section{Datos y metodología}

\subsection{Base de datos utilizada}

Como se ha señalado, no existen encuestas longitudinales para el conjunto de España que permitan abordar todo el conjunto de variables que consideramos relevantes para estudiar descriptiva y explicativamente los factores por los que ciertos colectivos viven situaciones de «atrapamiento» en el empleo inestable. Es por ello que, en esta investigación, se ha utilizado el Panel de Desigualdades $(\mathrm{PaD})$ de la Fundació Jaume Bofill (Fundació Jaume Bofill, 2012), realizado en Cataluña entre los años 2001 y $2012^{4}$. El PaD se fundamenta en una encuesta de tipo panel puro que se inició con una muestra de 5.757 personas de más de 16 años, y más de 2.000 hogares en Cataluña (Fundació Jaume Bofill, 2008). La base de datos utilizada ofrece información que no se encuentra en otras bases de datos de carácter longitudinal obtenidas a partir de registros administrativos. Ya se ha indicado anteriormente que estos registros administrativos no permiten identificar el origen social familiar, además de recoger de forma poco precisa otras variables relevantes como el nivel de estudios.

En nuestro análisis, se toman las cinco oleadas para el período 2007-2011, lo cual supone trabajar con una matriz inicial de 4.030 individuos para el año 2007. Se ha fijado el 2007 como el primer año de la serie analizada, porque, ya a finales de este año, empieza a crecer el desempleo entre la población ocupada, tras años de reducción progresiva. Se toma el año 2011 como final porque es el último en que se utilizó la misma metodología que en los años anteriores (encuesta en el hogar de los entrevistados mediante un cuestionario administrado cara a cara). Se obtiene, así, una serie de 5 años que permite analizar una buena parte del período de crisis del empleo en el cual nos encontramos.

\subsection{Análisis realizados y variables consideradas}

Con los datos indicados, se han realizado dos tipos de explotaciones. En primer lugar, un análisis de clasificación que ha permitido agrupar diferentes tipos de trayectorias para el período 2007-2011. Las trayectorias individuales que están en la base de este análisis descriptivo se han construido computando la presencia de situaciones de temporalidad, de desempleo, de inactividad y de mejora en la categoría laboral durante los cinco años considerados. Este análisis de clasificación se ha realizado inicialmente para todos los casos cuya edad se situaba, en el año 2011, entre los 20 y los 65 años $(1.139 \text { casos })^{5}$ y que, durante el período analizado, habían sido activos alguna vez en el mercado de trabajo $\mathrm{o}$, siendo inactivos, no estaban cursando estudios formales.

4. La crisis económica española no solo ha afectado a la situación de los trabajadores en el mercado de trabajo. Como consecuencia de la falta de fondos, la Fundació Jaume Bofill, responsable de la elaboración del Panel, elaboró la oleada correspondiente al año 2012 de modo telefónico y ya ha dejado de elaborarla en los años sucesivos.

5. En relación con su población de referencia, ello supone un error muestral del 2,9\% para un nivel de confianza del $95 \%$ y $p=q=0,5$. 
En el análisis, se incluye a las personas inactivas que no están estudiando porque consideramos que las trayectorias y los cursos vitales y laborales están condicionados por las diferentes distribuciones (negociadas o no) de los trabajos formales, informales y domésticos desarrollados en los núcleos de convivencia (Wallace, 2002). Esta distribución entre diferentes trabajos tiene un evidente sesgo de género, de manera que, si queremos tratar correctamente la influencia de esta variable a la hora de dar cuenta de los cursos laborales y vitales, es necesario incluir estas situaciones de inactividad. Así, los cursos vitales deben considerarse interrelacionados con los diferentes tipos de trabajo mencionados (Anxo et al., 2010), en que la participación continua en el mercado de trabajo formal de determinados perfiles se vincula a una participación menor o discontinua en relación con los otros trabajos, y viceversa. Por otro lado, se han eliminado los casos que son inactivos como consecuencia del hecho de estar cursando estudios formales, porque consideramos que el periodo de formación de estas personas no está aún finalizado, lo cual provoca distorsiones importantes al vincular el nivel máximo de estudios alcanzado con la situación en el mercado laboral.

Una vez obtenida la tipología de trayectorias de empleo para el conjunto de la muestra de 20 a 65 años, se ha procedido a comparar entre sí, mediante tablas de contingencia, la submuestra de entre 20 y 40 años y la submuestra de entre 41 y 65 años. Esta comparación permite comprobar el grado en que las trayectorias de los trabajadores que se insertaron laboralmente en un contexto de flexibilidad se caracterizan por la inestabilidad permanente, frente a las de aquellos que lo hicieron con anterioridad. Es decir, nos indicará hasta qué punto es posible hablar de la existencia de «polarización generacional»o no. Este análisis también permite situar, comparativa y contextualmente, en el conjunto de la población, las trayectorias de la submuestra de casos de entre 20 y 40 años, con la que posteriormente se ha realizado un análisis de carácter explicativo.

Situando la edad de corte en los 40 años, se obtiene una submuestra en que todos los individuos han desarrollado sus trayectorias laborales bajo un marco normativo e institucional común, caracterizado por un entorno de flexibilidad creciente y totalmente distinto del existente en el momento de la inserción laboral de aquellas cohortes de mayor edad. Ello no significa que demos por descontado que este nuevo marco no haya afectado a las generaciones anteriores. Precisamente, el análisis comparativo de los dos grupos de edad permite comprobar que, independientemente del contexto institucional en el momento de la inserción, durante los años de crisis, también se han desarrollado trayectorias inestables en una parte de la población de más de 41 años.

El segundo tipo de explotación estadística ha consistido en la elaboración de un modelo logístico multinominal, aplicado a la submuestra de casos de entre 20 y 40 años $(510 \text { casos })^{6}$. Este análisis ha tomando como variable depen-

6. En relación con su población de referencia, ello supone un error muestral del 4,3\% para un nivel de confianza del $95 \%$ y $p=q=0,5$. 
diente la que recoge los diferentes tipos de trayectorias identificados mediante el análisis de clasificación ${ }^{7}$. Como ya se ha indicado, con este análisis, se tratan de identificar los factores que podrían explicar el desarrollo de trayectorias divergentes entre individuos pertenecientes a las mismas cohortes.

Antes de realizar el modelo, se han analizado, mediante análisis de correlación y tablas de contingencia, las variables independientes edad, sexo, nivel de estudios, categoría laboral, sector, número de trabajadores en la empresa, tipo de organización (pública, privada, mixta, cooperativa, etc.), categoría socioeconómica del padre y de la madre y nivel de estudios del padre y de la madre. No se han incluido en el modelo las variables número de trabajadores ni tipo de organización, porque no mostraban una relación significativa con el hecho de estar en una u otra trayectoria, ni tampoco la categoría laboral del individuo, puesto que mostraba una colinealidad elevada con el nivel de estudios.

Por otro lado, se ha detectado una importante correlación entre el nivel de estudios del padre y de la madre (que, por otro lado, no se observa con el de los hijos e hijas), lo cual recomendaba no introducir ambas variables en el modelo. Nos hemos quedado con el nivel de estudios del padre, al resultar con una $\mathrm{R}^{2}$ más elevada. La introducción de las variables vinculadas al origen social permite aumentar la $\mathrm{R}^{2}$ de manera relevante respecto a otros modelos (que no presentamos) que no las incluyen, lo cual nos indica que estas variables permiten ganar un importante poder explicativo.

Es importante destacar que no resulta evidente el modo en que se puede introducir en las regresiones la información relativa al origen social como variable explicativa. La forma clásica de considerar esta información en relación con los logros educativos y laborales ha sido tomando la situación ocupacional y el nivel de estudios del padre, en lo que se ha llamado el modelo convencional (Goldthorpe, 1984). Esta perspectiva ha sido fuertemente criticada por la posición subordinada a la que relega a la mujer, por no responder a las dinámicas cambiantes del núcleo familiar ni del mercado de trabajo, y por no dar cuenta de la provisión del conjunto de recursos disponibles en el hogar o en la familia (Stanworth, 1984; Baxter, 1988). En base a estos análisis, se han desarrollado diferentes modelos, como aquellos que consideran el nivel de ocupación y estudios de nivel superior del padre o la madre (Eriksson, 1984), los que consideran la ocupación y el nivel de estudios del padre o la madre con nivel inferior de estudios u ocupación o aquellos que consideran al padre y a la madre en el mismo nivel. Desde este punto de vista, varios estudios empíricos (Korupp et al., 2002; Beller, 2009; Lampard, 2012) indican que los mejores resultados al explicar situaciones educativas y en el mercado de trabajo se obtienen al considerar la situación del padre y la madre, pues ambos son claves en definir el conjunto de recursos y capitales, tanto de índole económica como cultural, social o simbólica. Y es en relación a esta cuestión que todos estos autores

7. El escaso número de casos ha obligado a fusionar en una sola categoría dos de los grupos obtenidos mediante el análisis de clasificación. Véase el epígrafe 5.2 para una explicación más detallada. 
incluyen en el análisis a las madres dedicadas a las tareas del hogar consideradas como inactivas, puesto que mejoran los modelos explicativos de la situación de los hijos y de la movilidad social. Siguiendo estos argumentos, en nuestro análisis, hemos optado por introducir información relativa al padre y a la madre, siempre que no se diese colinealidad entre las variables.

\section{Resultados}

\subsection{Análisis descriptivo}

El análisis de conglomerados realizado para la población de 20 a 65 años ha permitido identificar cuatro tipos diferentes de trayectorias, que se diferencian por sus transiciones en el mercado de trabajo. Para realizar este análisis, se consideran los valores de las siguientes cuatro variables: frecuencias de desempleo, temporalidad, inactividad en el período 2007-2011 (que adquieren valores de 0 a 5 , en función de su presencia o ausencia en cada una de las observaciones anuales) y existencia de promoción laboral entre 2007 y 2011 (con valores de 0 y 1 , en función de si se observa un cambio positivo de situación o categoría laboral entre el inicio y el final del período ${ }^{8}$. En el análisis, se ha seguido el método Ward y se ha seleccionado la solución de cuatro conglomerados, al ser la que presentaba mayores diferencias entre los grupos. Esta selección se ha realizado en base a los valores obtenidos en los coeficientes de aglomeración y en las particiones observadas en el árbol de agregación y en el dendrograma de distancias. En la tabla 1, se presentan las medias y desviaciones típicas de las variables utilizadas en la elaboración de los conglomerados por cada trayectoria resultante, y en la tabla 2 , se observa la distribución de las trayectorias en relación con los dos grandes grupos de edad considerados (20-40 y 41-65).

Como se observa en la tabla 1, el tipo de trayectoria con mayor número de efectivos, que hemos denominado lineal, representa al 45\% de los casos y muestra una media de frecuencias de transiciones muy bajas. De hecho, las situaciones de desempleo e inactividad son inexistentes. Además, en esta trayectoria, se observa una proporción superior a la media ${ }^{9}$ de técnicos medios y superiores, así como de trabajadores administrativos; su salario se sitúa por encima de los 1.000 euros mensuales, con una proporción especialmente elevada de la franja salarial hasta los 2.000 euros. El siguiente grupo más numeroso, con el $25,2 \%$ de los casos, se ha denominado trayectoria de actividad discontinua y se caracteriza por una elevadísima frecuencia media de inactividad. Los datos muestran que se trata de trabajadores autónomos y también de trabajadores no cualificados; su elemento más significativo es el muy bajo salario que perciben, por debajo de los 600 euros mensuales. El siguiente grupo, con

8. En esta variable, se ha considerado igualmente como promoción el paso de situación de desempleo o de inactividad a cualquier situación de empleo.

9. Por cuestiones de espacio, no reproducimos aquí las tablas con datos de nivel salarial ni de categoría laboral. Los autores pondrán gustosamente estos datos a disposición de quien los demande. 
Tabla 1. Trayectorias laborales por frecuencia de transiciones (de 20 a 65 años). Cataluña 2007-2011

\begin{tabular}{llrrrr}
\hline Trayectorias & & $\begin{array}{c}\text { Frecuencia } \\
\text { de desempleo }\end{array}$ & $\begin{array}{l}\text { Frecuencia de } \\
\text { temporalidad }\end{array}$ & $\begin{array}{l}\text { Frecuencia } \\
\text { de inactividad }\end{array}$ & $\begin{array}{l}\text { Frecuencia de } \\
\text { aumento del nivel } \\
\text { de ocupación }\end{array}$ \\
\hline Precariedad & Media & 1,6778 & 0,2556 & 0,7000 & 0,3556 \\
$(15,8 \%)$ & Desv. típ. & 1,28008 & 0,47416 & 0,90868 & 0,52451 \\
& $N$ & 180 & 180 & 180 & 180 \\
\hline Lineal & Media & 0,0000 & 0,0838 & 0,0000 & 0,0526 \\
$(45 \%)$ & Desv. típ. & 0,00000 & 0,27739 & 0,00000 & 0,22351 \\
& $N$ & 513 & 513 & 513 & 513 \\
\hline Actividad & Media & 0,0697 & 0,0209 & 4,7213 & 0,0418 \\
discontinua & Desv. típ. & 0,25506 & 0,16593 & 0,61366 & 0,21725 \\
$(25,2 \%)$ & $N$ & 287 & 287 & 287 & 287 \\
\hline Temporalidad & Media & 0,4591 & 2,9245 & 0,1572 & 0,3711 \\
$(14 \%)$ & Desv. típ. & 0,74411 & 0,94499 & 0,41391 & 0,54603 \\
& $N$ & 159 & 159 & 159 & 159 \\
\hline Total & Media & 0,3468 & 0,4917 & 1,3222 & 0,1422 \\
$(100 \%)$ & Desv. típ. & 0,84055 & 1,08053 & 2,04964 & 0,37374 \\
& $N$ & 1139 & 1139 & 1139 & 1139 \\
\hline
\end{tabular}

Fuente: elaboración propia a partir del PaD (Fundació Bofill).

el 15,8\% de los casos, lo hemos denominado de precariedad, pues es el grupo con la media de desempleo más elevada en el período. Se trata de trabajadores manuales (cualificados y no cualificados) con un salario de hasta 1.000 euros al mes. Finalmente, el último grupo, con el $14 \%$ de los casos, se ha denominado de temporalidad; se caracteriza por la temporalidad, con una media muy superior a la del resto de grupos, y también una frecuencia de desempleo superior a la media del conjunto de la población, pero por debajo de la que presenta el grupo de precariedad. En este caso, la proporción de trabajadores no cualificados y de técnicos superiores está por encima de la media, y su salario se sitúa en los niveles de hasta 1.000 euros al mes. En estos dos últimos grupos más discontinuos, observamos frecuencias de aumento del nivel de ocupación superior a la media, en especial, en el grupo de temporalidad; se trata de individuos que provienen de categorías muy bajas y con un perfil de edad joven.

La tabla 2 nos permite comprobar que la selección de grupos de edad realizada en el artículo es fuertemente significativa en relación con las trayectorias. Si comparamos el peso que tiene cada tipo de trayectoria para cada uno de los dos grupos de edad, se observa una escasa proporción de personas de 20 a 40 años en la trayectoria de actividad discontinua, únicamente el 5,3\% de los casos. Se observa también una diferencia muy relevante en la trayectoria de temporalidad, que representa el 23,9\% del grupo de 20 a 40 años y solo el 5,9\% del grupo de 41 a 65 años. Igualmente, en la trayectoria lineal, se observan diferencias importantes, puesto que representa el $54,1 \%$ de las 
Tabla 2. Trayectorias por grupos de edad. Cataluña 2007-2011

\begin{tabular}{llrrr}
\hline Chi cuadrado & 228.021*** & $20-40$ & $41-65$ & Total \\
\hline Precariedad & $N$ & 85 & 95 & 180 \\
& $\%$ & $16,7 \%$ & $15,1 \%$ & $15,8 \%$ \\
& Residuos estandarizados & 0,2 & $-0,2$ & 513 \\
\hline Lineal & $N$ & 276 & 237 & $45,0 \%$ \\
& $\%$ & $54,1 \%$ & $37,7 \%$ & \\
\hline Actividad & $N$ & 3,2 & $-2,8$ & 287 \\
discontinua & $\%$ & 27 & 260 & $25,2 \%$ \\
& Residuos estandarizados & $5,3 \%$ & $41,3 \%$ & 159 \\
\hline Temporalidad & $N$ & $-8,9$ & 8,0 & $14,0 \%$ \\
& $\%$ & 122 & 37 & \\
\hline Total & Residuos estandarizados & $23,9 \%$ & $5,9 \%$ & 1.139 \\
& $N$ & 6,1 & $-5,5$ & $100 \%$ \\
\hline *** $P<0,01$ & $\%$ & 510 & 629 &
\end{tabular}

*** $p<0,01$

Fuente: elaboración propia a partir del PaD (Fundació Bofill).

trayectorias en el grupo de 20 a 40 años y solo el $37,7 \%$ en el grupo de 41 a 65 años. En cambio, la trayectoria de precariedad, que, como se ha señalado, es la que recoge las trayectorias con mayor presencia del desempleo, presenta unos porcentajes similares en los dos grupos de edad. Así, se comprueba que, entre los trabajadores más jóvenes, se da, en primer lugar, una mayor orientación hacia el mercado de trabajo, así como un porcentaje relevante de personas afectadas por la discontinuidad en forma de temporalidad. Si nos fijamos en los residuos estandarizados, identificamos que aquellas personas de hasta 40 años muestran una relación significativa con tener una trayectoria lineal o de temporalidad. En cambio, los de más de 40 años muestran una mayor relación con la de inactividad, lo cual provoca en buena medida su menor presencia en la trayectoria lineal.

Los valores de los residuos estandarizados de la tabla 2 muestran también que, durante el período 2007-2011, las trayectorias caracterizadas por el desempleo tienen una distribución estadísticamente no significativa entre la población por debajo de los 40 años y aquella que se sitúa por encima de esta edad. Así, en relación con la primera cuestión que nos planteábamos, relativa a la polarización de trayectorias entre generaciones, obtenemos una primera imagen que desmiente que, durante la crisis, sean los grupos de trabajadores más jóvenes los que hayan padecido las aquí denominadas trayectorias de precariedad. Las caracterizadas por el desempleo no constituyen una especificidad del colectivo más joven durante la crisis. Sin embargo, sí se puede afirmar que, durante el período 2007-2011, este colectivo ha padecido en mayor medida las situaciones de empleo temporal, algo que no es nuevo, 
como muestran los datos existentes para períodos anteriores y la propia revisión de la literatura.

Así pues, puede afirmarse que el grupo de edad insertado laboralmente durante el período posterior a las reformas flexibilizadoras del mercado de trabajo se muestra fuertemente afectado por la temporalidad en comparación con el colectivo de más edad. A pesar de esto, cerca del $50 \%$ ha mantenido una trayectoria lineal durante la crisis. Pero la tabla 2 no muestra una «polarización interna» dentro del grupo de menos de 40 años, sino que más bien se detecta una segmentación en tres bloques. Por un lado, frente a la trayectoria lineal, caracterizada por la continuidad, existe un segundo grupo afectado por la discontinuidad. Y, dentro de este segundo grupo, aparece un primer subgrupo afectado sobre todo por el desempleo (y la inactividad, si tenemos en cuenta la trayectoria de actividad discontinua) y un segundo subgrupo más afectado por la temporalidad. Los factores que pueden haber causado estas diferencias internas entre las trayectorias no lineales son abordados a continuación.

\subsection{Análisis explicativo}

Para llevar a cabo el modelo de regresión logística multinominal, y con el objetivo de identificar qué características individuales, de sector y de origen social influyen sobre el tipo de trayectoria laboral desarrollada entre los años 2007 y 2011, se ha agrupado la trayectoria de actividad discontinua con la de precariedad. Se han agrupado estos dos tipos de trayectorias para garantizar un número suficiente de grados de libertad en la regresión y por ser las que más se acercan en sus características (comprobadas con el análisis de conglomerados y el posterior análisis de tablas de contingencia). En el análisis de regresión, se consideran como variables independientes la edad (como variable continua), el género, el nivel de estudios, el sector de actividad, la categoría socioeconómica del padre y de la madre y el nivel de estudios del padre ${ }^{10}$.

La tabla 3 muestra que, frente a la trayectoria lineal, que es el valor de referencia y se caracteriza por la estabilidad, las trayectorias de discontinuidad están marcadas por factores significativamente diferentes. Así, una primera conclusión que se puede extraer de la tabla 3 es que las trayectorias caracterizadas por el «empleo de mala calidad» y el desempleo, representadas, por un lado, por la trayectoria de precariedad y actividad discontinua y, por otro, por la trayectoria de temporalidad, se corresponden con dos perfiles sociales con diferencias muy marcadas. La trayectoria de precariedad y actividad discontinua se caracteriza por mostrar como variables significativas buena parte de los factores ya conocidos que marcan el perfil de los trabajadores del segmento más precario en el mercado laboral español. El hecho de ser mujer y de tener una credencial educativa por debajo del nivel de licenciado distingue significativa-

10. Las variables número de trabajadores en la empresa, tipo de organización, categoría laboral y nivel de estudios de la madre no se han introducido por las razones explicadas en el apartado 4.2. 
Tabla 3. Modelo logístico multinominal. Influencia de las características individuales, el sector y el origen social en las trayectorias laborales (de 20 a 40 años). Cataluña 2007-2011

\begin{tabular}{|c|c|c|}
\hline $\begin{array}{l}\text { Referencia: } \\
\text { Trayectoria lineal }\end{array}$ & $\begin{array}{l}\text { Precariedad y actividad } \\
\text { discontinua }\end{array}$ & Temporalidad \\
\hline Edad & $-0,059$ & $-0,088^{\star \star \star}$ \\
\hline \multicolumn{3}{|l|}{ Género } \\
\hline Hombre & Ref. & Ref. \\
\hline Mujer & $0,976^{\star \star *}$ & 0,452 \\
\hline \multicolumn{3}{|l|}{ Nivel de estudios } \\
\hline Licenciados y estudios postuniversitarios & Ref. & Ref. \\
\hline Diplomatura & $2,477^{\star *}$ & 0,690 \\
\hline $\mathrm{FP}$ & $2,634^{\star *}$ & 0,141 \\
\hline Secundarios & $3,050^{\star \star \star}$ & 0,143 \\
\hline Hasta primarios & $3,129^{\star \star \star}$ & 0,735 \\
\hline \multicolumn{3}{|l|}{ Sector } \\
\hline Administración pública & Ref. & Ref. \\
\hline Primario & 1,863 & 0,475 \\
\hline Industria & $2,169^{* *}$ & 0,936 \\
\hline Construcción & $3,362^{\star \star \star}$ & $2,978^{\star \star \star}$ \\
\hline Servicios comerciales y mercantiles & 1,612 & 1,020 \\
\hline Educación, sanidad y servicios sociales & 1,411 & $1,621^{\star *}$ \\
\hline \multicolumn{3}{|l|}{ Categoría socioeconómica del padre } \\
\hline $\begin{array}{l}\text { Obreros manuales } \\
\text { (cualificados y no cualificados) }\end{array}$ & Ref. & Ref. \\
\hline $\begin{array}{l}\text { Cuadros, técnicos y empleados } \\
\text { no manuales }\end{array}$ & 0,406 & $0,845^{\star *}$ \\
\hline $\begin{array}{l}\text { Autónomos, propietarios de empresas sin } \\
\text { asalariados o con menos de } 10 \text { trabajadores }\end{array}$ & 0,383 & $-0,095$ \\
\hline $\begin{array}{l}\text { Directivos, empresarios } \\
\text { (de } 10 \text { o más trabajadores) y profesionales }\end{array}$ & $-0,178$ & 0,159 \\
\hline \multicolumn{3}{|l|}{ Nivel de estudios del padre } \\
\hline Universitarios & Ref. & Ref. \\
\hline Secundarios & 1,113 & 0,266 \\
\hline Hasta primarios & 0,945 & 0,001 \\
\hline \multicolumn{3}{|l|}{ Categoría socioeconómica de la madre } \\
\hline $\begin{array}{l}\text { Obreros manuales } \\
\text { (cualificados y no cualificados) }\end{array}$ & Ref. & Ref. \\
\hline Cuadros, técnicos y empleados no manuales & $-0,651$ & $-0,241$ \\
\hline $\begin{array}{l}\text { Autónomos, propietarios de empresas sin } \\
\text { asalariados o con menos de } 10 \text { trabajadores }\end{array}$ & $-0,693$ & $-1,194$ \\
\hline $\begin{array}{l}\text { Directivos, empresarios } \\
\text { (de } 10 \text { o más trabajadores) y profesionales }\end{array}$ & 0,527 & $-0,299$ \\
\hline Inactiva, ama de casa & $-0,212$ & $-1,103^{\star \star}$ \\
\hline Intersección & $-5,212^{\star \star \star}$ & 0,075 \\
\hline $\mathrm{R}^{2} \mathrm{McF}$ Fadden & $0,281^{\star \star \star}$ & \\
\hline$N$ & 389 & \\
\hline
\end{tabular}

Nota: ${ }^{\star \star} p<0,05 ;{ }^{\star \star \star} p<0,01$.

Fuente: elaboración propia a partir del PaD (Fundació Bofill). 
mente a este tipo de trayectoria, durante el período de crisis, de la trayectoria de temporalidad y de la lineal.

Por otro lado, la edad solo se asocia significativamente a la trayectoria de temporalidad, y no a las de precariedad y actividad discontinua. Ello nos hace pensar, apelando otra vez a las características de la estructura laboral española, que la trayectoria de precariedad y actividad discontinua es el reflejo longitudinal de aquellos trabajadores que quedan «atrapados» en los segmentos con mayor inestabilidad del mercado laboral, en que el paso de los años no permite conseguir la estabilidad aunque se acumule antigüedad o experiencia (de ahí la falta de significatividad de la variable edad).

Por el contrario, la edad significativamente más joven de las personas con trayectoria de temporalidad nos ofrece una clave interpretativa interesante respecto a los individuos que han desarrollado una trayectoria lineal. Así, esta diferencia puede leerse perfectamente en términos de efecto período: posiblemente, aquellos trabajadores que han entrado en el mercado de trabajo entre los años 2007 y 2011 se han encontrado con unas condiciones laborales peores que las obtenidas por los trabajadores con el mismo perfil (puesto que, en el resto de variables, su perfil es casi idéntico) que se insertaron con anterioridad a la crisis y que han desarrollado una trayectoria lineal. No disponemos de información sobre el momento de inserción en el mercado laboral de nuestros encuestados, por lo que solo la edad nos permite suponer que se trata de personas cuya inserción laboral se produjo con posterioridad a la de los trabajadores con trayectoria que hemos definido como lineal, pero otras fuentes de información también para Cataluña (Castelló et al., 2013) dan verosimilitud a esta interpretación. Por otro lado, tampoco podría descartarse totalmente un cierto efecto de la edad, puesto que, en el futuro, estos individuos más jóvenes, tras acumular antigüedad o experiencia, quizás empiecen a desarrollar trayectorias de mayor estabilidad, como las que han desarrollado sus homólogos de más edad entre los años 2007 a 2011. No obstante, tal como se ha indicado, y teniendo en cuenta la información contextual revisada en apartados anteriores, el efecto período parece tener mucha mayor incidencia que en el efecto edad.

Si tomamos en cuenta la influencia de las categorías vinculadas al sector, es interesante destacar que tanto el sector de la construcción como el industrial se vinculan significativamente al desarrollo de la trayectoria de precariedad y actividad discontinua. Se identifica aquí otro efecto importante del período de crisis económica: entre los años 2007 y 2011, la destrucción de empleo se ha concentrado tanto en el sector secundario como en la construcción (Recio, 2010), lo cual ha provocado trayectorias con elevada presencia del desempleo y la inactividad en los trabajadores de estos sectores. El sector de la construcción también se asocia a la trayectoria de temporalidad, así como también los de educación, sanidad y servicios sociales. Ello mostraría que, durante la crisis, en el primero de estos dos sectores, se ha combinado la destrucción de empleo con el uso de la contratación temporal, mientras que, en el segundo, ha predominado principalmente la segunda tendencia. 
En relación con el nivel educativo, debe destacarse que cualquier nivel por debajo del de licenciado se asocia al desarrollo de trayectorias de precariedady actividad discontinua. Por lo tanto, puede inferirse que la inversión en educación superior universitaria ha servido, durante la crisis económica, para evitar la trayectoria de mayor precariedad laboral, pero no para garantizar trayectorias de estabilidad. Como se comprueba, por tanto, se paga un alto "precio educativo" - como hemos visto que indican los estudios sobre la sobreeducación en España (Ortiz, 2010) - únicamente para evitar la trayectoria caracterizada por el desempleo y la inactividad.

Finalmente, en relación con la influencia del origen social, cabe destacar que no existen valores que diferencien significativamente la trayectoria de precariedad y actividad discontinua de la lineal. Como es conocido, el nivel de estudios está marcado en buena medida por el origen social (Carabaña, 2004; Albert y Davia, 2012), de modo que el acceso a la universidad es mucho más frecuente entre los hijos de categorías socioeconómicas medias y altas, pero estos datos parecen confirmar que el origen social por sí solo (una vez controlado el efecto de los estudios) no ejerce un efecto causal en el desarrollo de las trayectorias de precariedad y actividad discontinua.

Sin embargo, sí se hallan valores significativamente diferentes en relación con el origen social cuando se compara la trayectoria de temporalidad con la lineal. El hecho de no tener madres que sean inactivas (es decir, tener madres activas laboralmente) y de tener padres cuya categoría laboral sea cuadro, técnico o empleado no manual correlaciona positivamente con el desarrollo de trayectorias de temporalidad. Ello nos indica que tener un origen social vinculado con el perfil de clase media no propietaria y con un modelo familiar en que padre y madre gozan de una ocupación remunerada está asociado con el desarrollo de este tipo de trayectoria durante los años de crisis económica. Si recordamos que no existe ninguna diferencia significativa en el nivel de estudios de los individuos que han desarrollado la trayectoria de temporalidad y la lineal, podemos pensar que, en un contexto en que los recursos educativos no han sido suficientes para conseguir trayectorias laborables estables, se han movilizado otros recursos (capital económico familiar, redes de contactos) asociados a ciertos orígenes familiares (pequeños empresarios o autónomos, y directivos, empresarios o profesionales), con el objetivo de asegurar la estabilidad de las trayectorias.

\section{Conclusiones}

Con la explotación de los datos que se ha presentado, el artículo ha pretendido responder a dos preguntas vinculadas con el desarrollo de las trayectorias laborales de la población de menos de 40 años entre 2007 y 2011. En primer lugar, se quería comprobar si las trayectorias de la población menor de 40 años eran suficientemente diferentes (para mal) de las de la población de mayor edad como para afirmar que se da una «segmentación intergeneracional» durante el período de crisis económica. Adicionalmente, se quería comprobar el grado en que se ha dado una «segmentación intrageneracional» dentro de este mismo 
grupo de edad. En segundo lugar, se querían identificar aquellos factores que correlacionan significativamente con las diferentes trayectorias laborales desarrolladas por la población de menos de 40 años, y comprobar de este modo qué elementos permiten explicar el desarrollo de trayectorias laborales de «atrapamiento en la inestabilidad» durante la crisis económica.

Siendo conscientes de que la realidad económica de Cataluña no se corresponde exactamente con la de todo el conjunto de España, sí creemos que las conclusiones que se extraen del análisis pueden extrapolarse en buena medida, puesto que se comparte el entorno normativo, institucional e incluso social. Es por ello que, más que insistir en el volumen de personas afectadas por cada tipo de trayectoria o en su distribución porcentual, se ha hecho hincapié en las características de cada tipo de trayectoria y en los factores causales que se vinculan a ellas.

En relación con el primer objetivo anunciado, se ha comprobado que las personas que iniciaron su andadura laboral en un contexto marcado por la flexibilidad contractual no han desarrollado, en mayor proporción, y entre los años 2007 y 2011, trayectorias caracterizadas por la precariedad, frente a las desarrolladas por las personas cuya inserción laboral se produjo en un contexto institucional diferente. En este sentido, la «segmentación intergeneracional» no se daría en forma de polarización entre aquellas trayectorias estables y aquellas situadas en el extremo de la inestabilidad laboral, cuya característica más definitoria es su elevada frecuencia de desempleo. Sí puede afirmarse, en cambio, que las trayectorias caracterizadas por la temporalidad son muy poco frecuentes entre la población de más de 40 años.

Por otro lado, se ha comprobado que tampoco se ha dado, en el período estudiado, una polarización «intrageneracional» entre las personas pertenecientes a las cohortes más jóvenes. O por lo menos, y matizando la afirmación anterior, no se ha dado una polarización de trayectorias tal como se describe en la literatura para otros países de nuestro entorno económico, con un progresivo distanciamiento en las condiciones laborales de aquellos más formados respecto a las de aquellos menos formados. En el contexto español, parece darse más bien una segmentación de trayectorias en tres grupos, uno caracterizado por la estabilidad laboral, otro, por la temporalidad de los contratos y un tercero, por el desempleo y la inactividad.

En relación con el segundo objetivo, se ha comprobado que las trayectorias estables están protagonizadas, durante los años estudiados, por un perfil social no demasiado alejado del que corresponde a las trayectorias con fuerte temporalidad. Este segundo tipo de trayectorias están protagonizadas por trabajadores con un nivel de estudios que no es significativamente diferente del de los primeros. Sin embargo, tener una edad relativamente joven sí es un factor causal; también lo es trabajar en el sector de la construcción y en el de la educación, la sanidad y los servicios sociales, así como tener un origen social que se puede identificar con las clases medias urbanas no propietarias. Finalmente, en las trayectorias marcadas por el desempleo y la inestabilidad, el género y el nivel educativo (ser mujer o tener un nivel educativo por debajo de la licenciatura) son factores causales, así como el hecho de haber trabajado en el sector de la construcción o de la industria. 
Así pues, puede afirmarse que la crisis económica ha producido dos tipos de trayectorias laborales no lineales, cada una de ellas protagonizada por perfiles sociales diferentes. El género y el nivel de estudios siguen siendo variables discriminantes entre el desarrollo de trayectorias con los máximos niveles de precariedad e inestabilidad laboral y el desarrollo de trayectorias caracterizadas por el empleo temporal o, en el mejor de los casos, el empleo estable. A estos factores ya clásicos en el contexto español, hay que añadir la significatividad de la variable vinculada al sector de actividad. Por otro lado, en el contraste entre trayectorias caracterizadas por la temporalidad frente a las lineales, el nivel educativo no es significativamente diferente, pero sí lo es la edad y alguno de los valores asociados al origen social. El papel desempeñado por este último factor en los procesos de inserción y posterior promoción laboral en época de crisis exige el desarrollo de nuevas investigaciones.

Otra cuestión que aparece como relevante en el análisis realizado es el grado de clausura o porosidad entre los diferentes segmentos identificados. Los datos analizados permiten inferir que se ha dado un atrapamiento en las trayectorias más precarias del mercado de trabajo, aquellas en que predomina el desempleo, la inactividad, los bajos salarios y las ocupaciones manuales. Sin embargo, no puede sostenerse lo mismo respecto a las trayectorias caracterizadas por la temporalidad. $\mathrm{El}$ hecho de que los individuos con este tipo de trayectoria sean significativamente más jóvenes que aquellos con trayectoria lineal impide afirmar categóricamente que tal situación de estabilidad les resulte inalcanzable. Sin duda, la intensidad y la duración de la crisis están marcando este tipo de trayectorias, quizás de forma irreversible. Pero solo el paso del tiempo permitirá comprobar si, tras años de contratación temporal, se podrá llegar algún día a una situación de estabilidad contractual o si, por el contrario, la temporalidad (u otras situaciones de discontinuidad) habrá marcado el conjunto de la trayectoria laboral. Esta es una cuestión que también queda pendiente para futuras investigaciones, en que, con fortuna, se podrá disponer de series temporales con un mayor recorrido temporal.

\section{Referencias bibliográficas}

Albert, Cecilia y Davia, María Ángeles (2012). «Trayectorias y logros educativos tras la Educación Secundaria Obligatoria (ESO)». Presupuesto y Gasto Público, 67, 161-176.

Alonso, Luis Enrique y Fernández, Carlos Jesús (2008). «Jóvenes: precariedad laboral, precariedad de vida». Gaceta Sindical: Reflexión y Debate, 10, 67-84.

Anxo, Dominique; Bosch, Gerhard y Rubery, Jill (2010). «Shaping the life course: A European perspective». En: Anxo, D.; Bosch, G. y Rubery, J. (eds.). The Welfare State and Life Transitions: A European Perspective. Cheltenham, UK: Edward Elgar, 1-77.

Autor, David H. (2014). «Skills, education, and the rise of earnings inequality among the "other 99 percent"». Science [en línea], 344 (6186), 843-851. $<$ http://dx.doi.org/10.1126/science.1251868>.

Banyuls, Josep y Recio, Albert (2012). "Spain: the nightmare of Mediterranean neoliberalism». En: LeHndorfF, Steffen (ed.). A triumph of failed ideas: European models of capitalism in the crisis. Bruselas: ETUI, 199-217. 
Banyuls, Josep; Miguélez, Fausto; Recio, Albert; Cano, Enric y Lorente, Raúl (2009). "The transformation of the employment system in Spain: Towards a Mediterranean Neoliberalism?». En: Bosch, G.; Lehndorff, S. y Rubery J. (eds.). European Employment Models in Flux: A Comparison of Institutional Change in Nine European Countries. Nueva York: Palgrave-Macmillan, 247-269.

BAXTER, Janeen (1988). "Gender and class analysis: The position of women in the class structure». Journal of Sociology [en línea], 24, 106-123. <http://dx.doi.org/10.1177/144078338802400106>.

Bell, David N.F. y Blanchflower, David G. (2011). "Young people and the Great Recession». Oxford Review of Economic Policy [en línea], 27 (2), 241-267. $<$ http://dx.doi.org/10.1093/oxrep/grr011>.

BeLler, Emily (2009). «Bringing intergenerational social mobility research into the twenty-first century: Why mothers matter». American Sociological Review [en línea], 74, 507-528. <http://dx.doi.org/10.1177/000312240907400401>.

Bernardi, Fabrizio y Garrido, Luis (2008). «Is there a new service proletariat?: Post-industrial employment growth and social inequalities in Spain». European Sociological Review [en línea], 24 (3), 299-313. <http://dx.doi.org/10.1093/esr/jcn003>.

Bernardi, Fabrizio y Martínez-Pastor, Juan Ignacio (2010). «Falling at the bottom: Unskilled jobs at entry in the labour market in Spain over time and in a comparative perspective». International Journal of Comparative Sociology [en línea], 51 (4), 289-307. <http://dx.doi.org/10.1177/0020715210368841>.

Berton, Fabio; Devicienti, Francesco y Pacelli, Lia (2011). «Are temporary jobs a port of entry into permanent employment?: Evidence from matched employer-employee». International Journal of Manpower [en línea], 32 (8), 879-899. <http://dx.doi.org/10.1108/01437721111181651>.

Bonhomme, Stéphane y Hospido, Laura (2013). «Earnings inequality in Spain: New evidence using tax data». Applied Economics [en línea], 45 (30), 4212-4225. <http://dx.doi.org/10.1080/00036846.2013.781261>.

Brueckner, Hannah y Mayer, Karl-Ulrich (2005). «De-standardization of the life course: What it might mean? And if it means anything, whether it actually took place?». Advances in Life Course Research [en línea], 9, 27-53. <http://dx.doi.org/10.1016/s1040-2608(04)09002-1>.

Bruno, Giovanni S.F.; Caroleo, Floro E. y Dessy, Orietta (2012). Stepping Stones versus Dead End Jobs: Exits from Temporary Contracts in Italy after the 2003 Reform [en línea]. IZA Discussion Paper número 6746. <http://hdl.handle.net/>.

CACHón, Lorenzo (2008). «De las políticas de transición en Europa a las clases de transiciones y transiciones de clase en España». Pensamiento Iberoamericano, 3, 97-116.

CARABAÑA, Julio (1997). "Educación y estrategias familiares de reproducción». En: Garrido Medina, L. y Gil Calvo, E. (ed.). Estrategias familiares. Madrid: Alianza, 37-47.

- (2004). «Educación y movilidad social». En: Navarro, V. (coord.). El Estado de Bienestar en España. Barcelona: Tecnos-UPF, 209-237.

Carrasco, Raquel; Jimeno, Juan F. y Ortega, A. Carolina (2014). «Returns to skills and the distribution of wages: Spain 1995-2010». Oxford Bulletin of Economics and Statistics [en línea], 77 (4), 542-565. <http://dx.doi.org/10.1111/obes.12077>. 
Casal, Joaquim; García, Maribel; Merino, Rafael y Quesada, Miguel (2006). "Changes in forms of transition in contexts of informational capitalism». Papers: Revista de Sociologia [en línea], 79, 195-223.

Castelló, Laia; Bolíbar, Mireia; Barranco, Oriol y Verd, Joan Miquel (2013). "Treball: Condicions en el mercat de treball i trajectòries laborals de la joventut catalana». En: Serracant, Pau (coord.). Enquesta de la Joventut de Catalunya 2012. Volum 1. Transicions juvenils $i$ condicions materials d'existència. Barcelona: Generalitat de Catalunya. Direcció General de Joventut, 117-224.

Castillo, Juan José y López Calle, Pablo (2007). «Una generación esquilmada: Los efectos de las Reformas Laborales en la vida y trabajo de los jóvenes madrileños». Sociedad y Utopia: Revista de Ciencias Sociales, 29, 293-311.

Cebrián, Inmaculada (2008). «Empleo temporal e indefinido y trayectorias laborales». Gaceta Sindical: Reflexión y Debate, 10, 43-66.

CEDefor (2011). Labour-market polarisation and elementary occupations in Europe: Blip or long term trend? Research Paper, 9. Luxemburgo: Publications Office of the European Union.

De Lange, Marloes; Gesthuizen, Maurice y Wolbers, Maarten H.J. (2014). "Youth labour market integration across Europe: The impact of structural, cyclical and institutional characteristics». European Societies [en línea], 16, 194-212. <http://dx.doi.org/10.1080/14616696.2013.821621>.

Doeringer, Peter y Piore, Michael (1971). Internal Labor Markets and Manpower Analysis. Lexington, DC: Heath and Co.

EDwards, Richard C. (1975). "The social relations of production in the firm and labour market structure». Politics \& Society [en línea], 5, 83-108. <http://dx.doi.org/10.1177/003232927500500104>.

Emmenegger, Patrick; Häusermann, Silja; Palier, Bruno y Seeleib-Kaiser, Martin (2012). «How we grow unequal». En: Emmenegger, Patrick; Häusermann, Silja; Palier, Bruno y Seeleib-Kaiser, Martin (eds.). The Age of Dualization: The Changing Face of Inequality in Deindustrializing Societies. Oxford: Oxford University Press, 3-26.

Eriksson, Robert (1984). «Social Class of Men, Women and Families». Sociology [en línea], 18, 500-514. <http://dx.doi.org/10.1177/0038038584018004003>.

Eurofound (2013). Employment polarization and job quality in the crisis: European Jobs Monitor 2013. Thessaloniki: European Foundation for the Improvement of Living and Working Conditions.

Fenton, Steve y Dermott, Esther (2006). «Fragmented careers?: Winners and losers in young adult labour markets». Work, Employment and Society [en línea], 20 (2), 205-221. <http://dx.doi.org/10.1177/0950017006064111>.

Ferreiro, Jesús; BeA, Eva; Gómez, M. Carmen y Intxausti, M. Ángeles (2004). "Teoría Insider-Outsider y temporalidad en el mercado de trabajo español». Revista del Ministerio de Trabajo y Asuntos Sociales, 51, 31-53.

Fundació Jaume Bofill (2008). El Panel de Desigualtats Socials a Catalunya (PaD): Apunts metodològics de l'enquesta. Barcelona: Mediterrània.

Fundació Jaume Bofill (2012). Enquesta panel de desigualtats socials a Catalunya. 20012012. Barcelona: Fundació Jaume Bofill [documento en línea]. <http://obdesigualtats.cat/intra/ob/documents/DOSSIER\%20PaD_WEB\%2020_02_12.pdf>

Fundación CYD (2014). Informe CYD 2013. Barcelona: Fundación Conocimiento y Desarrollo. 
García-Montalvo, José (2008). «La dinámica de la sobrecualificación en España». En: IVIE (ed.). Análisis de la sobrecualificación y la flexibilidad laboral: Observatorio de la inserción laboral de los jóvenes 2008. Valencia: Fundación Bancaja, 21-88.

García-Pérez, J. Ignacio y Muñoz-Bullón, Fernando (2011). «Transitions into Permanent Employment in Spain: An Empirical Analysis for Young Workers». British Journal of Industrial Relations [en línea], 49 (1), 103-143. <http://dx.doi.org/10.1111/j.1467-8543.2009.00750.x>.

Genda, Yuji; Kondo, Ayako y OHta, Souichi (2010). «Long-Term Effects of a Recession at Labor Market Entry in Japan and the United States». Journal of Human Resources [en línea], 45 (1), 157-196. <http://dx.doi.org/10.1353/jhr.2010.0005>.

Goldthorpe, John (1984). "Women and class analysis: a reply to the replies». Sociology [en línea], 18 (4), 491-499. <http://dx.doi.org/10.1177/0038038584018004002>.

Goos, Maarten; Manning, Alan y Salomons, Anna (2009). "Job polarization in Europe». American Economic Review [en línea], 99 (2), 58-63. <http://dx.doi.org/10.1257/aer.99.2.58>.

Häusermann, Silja y Schwander, Hanna (2012). «Varieties of dualization?: Labor Market Segmentation and Insider-Outsider Divides across regimes». En: EMMEnegger, Patrick; Häusermann, Silja; Palier, Bruno y Seeleib-Kaiser, Martin (eds.). The Age of Dualization: The Changing Face of Inequality in Deindustrializing Societies. Oxford: Oxford University Press, 27-51.

Hernanz, Virginia (2003). El trabajo temporal y la segmentación: Un estudio de las transiciones laborales. Madrid: Consejo Económico y Social.

Heyes, Jason (2014). Vocational education and training and the Great Recession: Supporting young people in a time of crisis. Bruselas: ETUI.

Hillmert, Steffen (2011). "Occupational mobility and developments of Inequality along the life course». European Societies [en línea], 13 (3), 401-423. <http://dx.doi.org/10.1080/14616696.2011.568263>.

ILO (2011). Spain: Quality jobs for a new economy. Ginebra: ILO Publications.

Kelly, Elish; McGuinness, Seamus y O’Connell, Philip J. (2012). «Transitions to long-term unemployment risk among young people: Evidence from Ireland». Journal of Youth Studies [en línea], 15 (6), 780-801. <http://dx.doi.org/10.1080/13676261.2012.678047>.

Косн, Max (2006). Roads to Post-Fordism: Labour Markets and Social Structures in Europe. Aldershot: Ashgate.

Korupp, Sylvia E.; Ganzeboom, Harry B.G. y Van Der Lippe, Tanja (2002). «Do Mothers Matter?: A Comparison of Models of the Influence of Mothers' and Fathers' Educational and Occupational Status on Childrens' Educational Attaintment». Quality \& Quantity [en línea], 36, 17-42. <http://dx.doi.org/10.1023/A:1014393223522>.

Kwon, Illong; Milgrom, Eva Meyersson y Hwang, Seiwoon (2010). «Cohort Effects in Promotions and Wages: Evidence from Sweden and the United States». Journal of Human Resources [en línea], 45 (3), 772-808. <http://dx.doi.org/10.1353/jhr.2010.0017>.

LAMPARD, Richard (2012). «Parental Characteristics, Family Structure and Occupational Attaintment in Britain». Sociology [en línea], 46 (6), 1020-1038. <http://dx.doi.org/10.1177/0038038511435062>. 
Lehwess-Litzmann, René (2014). Capability as a Yardstick for Flexicurity. Gottingen: Universitätsverlag Göttingen.

Lindbeck, Assar y SnOwer, Dennis J. (2001). «Insiders versus outsiders». Journal of Economic Perspectives, 15 (1), 165-188.

Martín, Antonio y Gibert, Francesc (2006). "The case of Spain: Growing and Secondary Labour Markets». En: KöHler, C.; Junge, K.; Schröder, T. y STruck, O. (eds.). Trends in employment stability and labour market segmentation. Jena: Friedrich-Schiller Universität Jena.

Martínez Celorrio, Xavier y Marín, Antoni (2010). Educació i ascens social a Catalunya. Barcelona: Fundació Jaume Bofill.

Mills, Melinda; Blossfeld, Hans-Peter; Buchholz, Sandra; Hofächer, Dirk; Bernardi, Fabrizio y Hofmaister, Heather (2008). «Converging Divergences?: An International Comparison of the Impact of Globalization on Industrial Relations and Employment Careers». International Sociology [en línea], 23 (4), 561-595. <http://dx.doi.org/10.1177/0268580908090728>.

Moreno-Galbis, Eva y Sopraseuth, Thepthida (2014). "Job polarization in aging economies». Labour Economics [en línea], 27, 44-55. <http://dx.doi.org/10.1016/j.labeco.2013.12.001>.

Muñoz de Bustillo, Rafael y Antón, José-Ignacio (2012). «From the highest employment growth to the deepest fall: Economic crisis and labour inequalities in Spain». En: Vaughan-Whitehead, D. (ed.). Inequalities in the world of work: The effects of the crisis. Ginebra: ILO, 397-448.

Navarro, José (2013). Universidad y mercado de trabajo en Cataluña: Un análisis de la inserción laboral de los titulados universitarios. Universidad Autónoma de Barcelona. Tesis doctoral.

Oesch, Daniel y Rodriguez, Jorge (2010). «Upgrading or polarization?: Occupational change in Britain, Germany, Spain and Switzerland, 1990-2008». Socioeconomic Review [en línea], 9 (3), 503-531. $<$ http://dx.doi.org/10.1093/ser/mwq029>.

Ortiz, Luis (2010). «Not the right job, but a secure one: Over-education and temporary employment in France, Italy and Spain». Work, Employment and Society [en línea], 24 (1), 47-64. <http://dx.doi.org/10.1177/0950017009353657>.

Ortiz, Isabel y Cummins, Matthew (2013). The Age of Austerity: A Review of Public Expenditures and Adjustement Measures in 181 Countries [en línea]. Initiative for Policy Dialogue and the South Centre Working Paper. Nueva York; Ginebra: Initiative for Policy Dialogue / The South Centre. <http://ssrn.com/abstract=2260771>, <http://dx.doi.org/10.2139/ssrn.2260771>.

Prieto, Alfonso (2012). «La segmentación del mercado de trabajo en España: Un análisis de su evolución en la crisis». Cuadernos del Mercado de Trabajo, $8,20-25$.

Recio, Albert (1997). Trabajo, personas, mercados. Barcelona/Madrid: Icaria-FUHEM.

- (2009). "La situación laboral de los jóvenes». Arquitectura, Ciudad y Entorno, 5, 411-426.

- (2010). "Capitalismo español: La inevitable crisis de un modelo insostenible». Revista de Economía Crítica, 9, 198-222.

Rocha, Fernando (2012). Youth Unemployment in Spain: Situation and Policy Recommendations. Berlín: Friedrich-Ebert-Stiftung. 
Rocha, Fernando y Negueruela, Enrique (2014). El mercado de trabajo en España en 2013: ¿Hacia una recuperación frágil y socialmente injusta de la crisis? Madrid: Fundación $10^{\circ}$ de Mayo.

SAnTAMAría López, Elsa (2012). "Jóvenes y precariedad laboral: Trayectorias laborales por los márgenes del empleo». Zerbitzuan: Gizarte Zerbitzuetarako Aldizkaria I Revista de Servicios Sociales [en línea], 52, 129-139. <http://dx.doi.org/10.5569/1134-7147.52.08>.

Santos, Antonio (2006). "Generación flexible": Vivencias de flexibilidad de los jóvenes parados». Cuadernos de Relaciones Laborales, 24 (2), 63-83.

Santos, Antonio y Martín, Paz (2012) «La juventud española en tiempos de crisis: Paro, vidas precarias y acción colectiva». Sociología del Trabajo, 75, 93-110.

Scarpetta, Stefano; Sonnet, Anne y Manfredi, Thomas (2010). Rising Youth Unemployment During the Crisis: How to Prevent Negative Long-term Consequences on a Generation? [en línea]. OECD Social, Employment and Migration Working Papers, 106. París: OECD Publishing. <http://dx.doi.org/10.1787/5kmh79zb2mmv-en>.

Scherer, Stefani (2004). «Stepping-Stones or Traps?: The Consequences of Labour Market Entry Positions on Future Careers in West Germany, Great Britain and Italy». Work, Employment and Society [en línea], 18 (2), 369-394. <http://dx.doi.org/10.1177/09500172004042774>.

Serracant, Pau (2010). «I com ho viu la gent jove?». En: Subirats, Marina (coord.). Societat Catalana. 2010. Barcelona: Institut d'Estudis Catalans. Associació Catalana de Sociologia.

Simó, Carles (2008). Les transicions a la vida adulta en el context de la globalització. Barcelona: Secretaria de Joventut. Generalitat de Catalunya.

SKans, Oskar Nordström (2011). Scarring effects of the first labor market experience [en línea]. Discussion paper series / Forschungsinstitut zur Zukunft der Arbeit, 5565. <http://hdl.handle.net/10419/51743>.

Stanworth, Michelle (1984). «Women and Class Analysis: A Reply to John Goldthrope». Sociology [en línea], 18, 159-170. <http://dx.doi.org/10.1177/0038038584018002001>.

Toharia, Luis; Davia, María Ángeles y Hernanz, Virginia (2001). Flexibilidad, juventud y trayectorias laborales en el mercado de trabajo. Madrid: CIS.

Tomlinson, Mark y Walker, Robert (2012). "Labor market disadvantage and the experience of recurrent poverty». En: Emmenegger, Patrick; Häusermann, Silja; Palier, Bruno y Seeleib-Kaiser, Martin (eds.). The Age of Dualization: The Changing Face of Inequality in Deindustrializing Societies. Oxford: Oxford University Press, 52-70.

Verd, Joan Miquel y López-Andreu, Martí (2012). «La inestabilidad del empleo en las trayectorias laborales: Un análisis cuantitativo». Revista Española de Investigaciones Sociológicas [en línea], 138, 135-148. <http://dx.doi.org/10.5477/cis/reis.138.135>.

Wallace, C.D. (2002). «Household strategies: Their conceptual relevance and analytical scope in social research». Sociology [en línea], 36 (2), 279-292. <http://dx.doi.org/10.1177/0038038502036002003>. 